\title{
Identification of candidate genes involved in coronary artery calcification by transcriptome sequencing of cell lines
}

\author{
Shurjo K Sen ${ }^{1}$, Jennifer J Barb², Praveen F Cherukuri ${ }^{1}$, David S Accame ${ }^{1}$, Abdel G Elkahloun ${ }^{1}$, Larry N Singh ${ }^{1}$, \\ Shih-Queen Lee-Lin', NISC Comparative Sequencing Program, Frank D Kolodgie ${ }^{3}$, Qi Cheng ${ }^{3}$, XiaoQing Zhao ${ }^{3}$, \\ Marcus Y Chen ${ }^{4}$, Andrew E Arai ${ }^{4}$, Eric D Green ${ }^{1}$, James C Mullikin ${ }^{1}$, Peter J Munson ${ }^{2}$ and Leslie G Biesecker ${ }^{1 *}$
}

\begin{abstract}
Background: Massively-parallel cDNA sequencing (RNA-Seq) is a new technique that holds great promise for cardiovascular genomics. Here, we used RNA-Seq to study the transcriptomes of matched coronary artery disease cases and controls in the ClinSeq ${ }^{\oplus}$ study, using cell lines as tissue surrogates.

Results: Lymphoblastoid cell lines (LCLs) from 16 cases and controls representing phenotypic extremes for coronary calcification were cultured and analyzed using RNA-Seq. All cell lines were then independently re-cultured and along with another set of 16 independent cases and controls, were profiled with Affymetrix microarrays to perform a technical validation of the RNA-Seq results. Statistically significant changes $(p<0.05)$ were detected in 186 transcripts, many of which are expressed at extremely low levels (5-10 copies/cell), which we confirmed through a separate spike-in control RNA-Seq experiment. Next, by fitting a linear model to exon-level RNA-Seq read counts, we detected signals of alternative splicing in 18 transcripts. Finally, we used the RNA-Seq data to identify differential expression $(p<0.0001)$ in eight previously unannotated regions that may represent novel transcripts. Overall, differentially expressed genes showed strong enrichment $(p=0.0002)$ for prior association with cardiovascular disease. At the network level, we found evidence for perturbation in pathways involving both cardiovascular system development and function as well as lipid metabolism.

Conclusions: We present a pilot study for transcriptome involvement in coronary artery calcification and demonstrate how RNA-Seq analyses using LCLs as a tissue surrogate may yield fruitful results in a clinical sequencing project. In addition to canonical gene expression, we present candidate variants from alternative splicing and novel transcript detection, which have been unexplored in the context of this disease.
\end{abstract}

Keywords: Coronary artery calcification, RNA-Seq, Lymphoblastoid cell lines, Transcriptome profiling

\section{Background}

Coronary Artery Disease (CAD) is a leading cause of mortality worldwide. The etiology of CAD is influenced by lifestyle, genetics, and gut microbiome [1,2] although the balance of these contributors remains unclear. While numerous GWAS projects have explored the genetics of CAD susceptibility [3], comparatively less is known about gene expression changes in this disease [4]. This is

\footnotetext{
* Correspondence: lesb@mail.nih.gov

${ }^{1}$ National Human Genome Research Institute, National Institutes of Health, Bethesda, MD 20892, USA

Full list of author information is available at the end of the article
}

partly because CAD is a complex phenotype involving multiple physiological systems. Consequently, no single tissue may fully reflect the network of gene expression changes underlying the disease [5].

In addition, current knowledge of the CAD transcriptome is based on gene expression microarrays, a technology that is useful but has several limitations. Recently, RNA-Seq, a powerful new technique for transcriptome analysis has revolutionized gene expression analyses by providing the ability to simultaneously interrogate all transcripts in an RNA sample (unlike microarrays which are limited to previously annotated transcripts) [6]. In addition, the high resolution

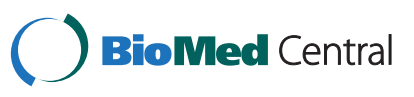


of RNA-Seq can facilitate discoveries of transcript dysregulation that have been missed by previous technologies. Here, we present results from a pilot application of RNA-Seq on human cases and controls chosen to reflect extremes for coronary artery calcification (CAC), a clinical marker for advanced CAD that is highly correlated with future adverse cardiovascular events [7]. As a patient surrogate for gene expression, we used EpsteinBarr virus transformed lymphoblastoid cell lines (LCLs), which have been shown in multiple studies to reliably reflect gene expression signatures [8], particularly those associated with nearby cis-acting genomic polymorphisms (expression quantitative trait loci or eQTLs) [9].

Here, we used RNA-Seq to compare LCLs from matched CAC cases and controls in the ClinSeq ${ }^{\circledR}$ project [10], and report a set of candidate genes for CAD that show differential expression and splicing variation between the groups, as well as novel transcripts that may have relevance for disease. RNA-Seq results were experimentally and technically replicated by repeating the cell culture part of our experiments and then measuring gene expression independently using expression microarrays. At the network level, we demonstrate that these differentially expressed genes are enriched for prior CAD association as well as for biological pathways with direct relevance to atherosclerosis.

\section{Methods}

An expanded Methods section is provided in Additional file 1.

\section{Coronary calcification scoring}

CAC scoring was performed using multi-slice computed tomography (Aquilion One, Toshiba Medical Systems, Japan or Lightspeed VCT, General Electric Healthcare, Waukesha, Wisconsin) with the use of prospective electrocardiographic gating. Calcification was quantified using the Agatston scoring method [11] on a dedicated workstation (VitreaFX or GE Advantage). The study was approved by the NHGRI Institutional Review Board and all subjects gave written informed consent.

\section{Cell culture, RNA-Seq library preparation, and sequencing}

LCLs were established by Epstein-Barr Virus (EBV) inoculation of B-lymphocytes using standard procedures, grown to a density of $2-3 \times 10^{5}$ cells $/ \mathrm{ml}$, harvested when the culture reached $10^{7}$ cells in total (5-6 passages) and stored at $-80^{\circ} \mathrm{C}$. Total RNA from $4 \times 10^{6}$ cells was extracted using the RNeasy Mini kit (Qiagen). RNA integrity was assessed using an Agilent Bioanalyzer (Agilent). RNA-Seq libraries were constructed using a custom protocol (see Additional file 1). Sequencing was done on Illumina $\mathrm{GAII}_{\mathrm{x}}$ sequencers (Illumina), collecting two lanes of $51 \mathrm{bp}$ reads for each library.

\section{RNA-Seq data processing}

Reads were aligned to the human genome (hg18) using TopHat [12]. The TopHat output SAM file was converted to BED format using the bamToBed script from the BEDTools package [13]. The coverageBed script from BEDTools was used to count reads mapping to individual exons in the RefSeq database (see Additional file 1). A total count for each transcript in this database was obtained by adding the counts for its constituent exons. These counts, after filtering and normalization (see Additional file 1), were used for statistical analysis of differential expression. We confirmed the subject identity in all RNA-Seq libraries by genotyping a subset of expressed SNPs from the corresponding genomic DNA. This indicated some contamination in the data for subject 133871; hence, this subject was excluded from further RNA-Seq analyses. The transcript-level RNA-Seq counts were first analyzed using a two level, one-way ANOVA to compare the expression in the case and control groups. Concurrently, the count data were analyzed using the edgeR Bioconductor package with default settings using the moderated tagwise dispersion option and the prior. $\mathrm{N}$ parameter set to 4 .

\section{Affymetrix exon array experiment}

For each sample, two micrograms of total RNA were used in conjunction with the Whole-Transcript Expression Analysis protocol for Affymetrix Human Exon 1.0 ST arrays. Arrays were scanned using an Affymetrix Gene Chip Scanner 3000 and intensities were calculated using Affymetrix AGCC software. RMA (Robust Multichip Average) signal intensities were calculated using Affymetrix Expression Console, converted to arithmetic scale, and transformed using an adaptive variance-stabilizing, quantile-normalizing transformation that was scaled to match the transformation used on the RNA-Seq data. A two level, one-way ANOVA was run on the microarray expression results.

\section{Western blot analysis of IGLL5}

A small piece of human carotid atherosclerotic plaque was frozen in liquid nitrogen and crushed to fine powder using an alloy tool steel mortar and pestle set that was pre-cooled in liquid nitrogen. The tissue powder was transferred to an ice-cold microcentrifuge tube and icecold cell lysis buffer (RayBiotech \#0103004-L) containing protease inhibitor cocktail (Sigma P8430) was added; the tissue weight to buffer volume ratio was $0.1 \mathrm{~g} / 0.3 \mathrm{ml}$. The samples were vortexed for 20 seconds at 10-minute intervals for 30 mins (and were kept on ice when not being vortexed). Samples were then centrifuged at $14000 \mathrm{~g}$ for 15 minutes at $4^{\circ} \mathrm{C}$; the supernatant was collected in new microcentrifuge tubes. Protein concentrations in the supernatant were quantified by using Micro BCA 
Protein Assay Kit (Pierce \#23225) according to manufacturer's instructions. 50ug of protein from each sample was separated on a 4 - 20\% polyacrylamide gel (Bio-Rad, \#1611159) with Tris-glycine - SDS buffer (Bio-Rad, \#161-0732). The proteins were transferred onto Immuno-Blot PVDF membranes (Bio-Rad, \#162-0174). The membrane was blocked with $5 \%$ non-fat dry milk in Tris-buffered saline Tween $20(0.5 \%)$ for 1 hour at room temperature, then incubated with primary antibody (anti human IGLL5, Abgent \#AP18459b) at a concentration of 1:200 overnight at $4^{\circ} \mathrm{C}$. The membranes were washed with Trisbuffered saline Tween 20 (0.5\%) three times, incubated with a secondary peroxidase - linked antibody (anti rabbit IgG HRP conjugate, Bio-Rad \#170-6515) for two hours at room temperature and then washed three times with Tris-buffered saline Tween 20 (0.5\%). The reactive bands were visualized by a chemiluminescence kit (Bio-Rad, \#170-5040) on Kodak BioMax Light film. The intensity of each IGLL5 band was analyzed by using the Chemidoc XRS system with Western blot analysis software (Bio-Rad). The final intensity was adjusted by individual loading control intensity readings (beta-actin).

\section{Detection of novel transcripts}

The Ensembl, AceView, ccDs, knownGenes, refGene, tRNA and rnaGene annotation tables were downloaded in GTF format from the UCSC Genome Browser (http:// www.genome.ucsc.edu) and combined into one master annotation file using the Cuffmerge tool in Cufflinks [14]. Transcripts were assembled using RNA-Seq data from a separate LCL library with extremely high depth of sequencing ( 200 million read pairs) using Cufflinks with the $-\mathrm{M}$ option to mask transcripts in the combined annotation file. These putative novel transcripts were then used as a reference annotation to run Cuffdiff, with the same BAM files from the case and control subjects that were used for quantifying gene expression. Loci detected as significant after Benjamini-Hochberg multiple testing correction were visually inspected by viewing the raw sequencing data (in BAM and BigWig format) on the UCSC Genome Browser with all gene and gene prediction tracks (and the ENCODE gene regulation track) turned on.

\section{Detection of alternatively spliced transcripts}

For alternative splicing, the exon-level counts were fit to a linear mixed-effect three-factor ANOVA model containing the following terms:

$$
y_{i j k}=\mu+A_{i}+\beta_{j(i)}+C+A C_{i k}+\varepsilon_{i j k}
$$

where $y$ was the normalized read count for an exon, $A_{i}$ was the fixed treatment effect for 1 through $i$ treatments (in this case, the case or control status), $\beta_{j(i)}$ was the random sample effect for sample $j$ within treatment, $C_{k}$ was the fixed exon effect for 1 through $k$ exons within a transcript, $A C_{i k}$ was the fixed interaction "treatment X exon" effect and $\varepsilon$ was the error factor. The ANOVA p-value for $\mathrm{p}-A C$ (which indicates the strength of the exon-treatment interaction) was then used to select for exons showing significantly different usage between cases and controls. Independently, the cuffdiff algorithm [14] was also used to detect alternatively spliced transcripts.

\section{Results}

\section{Assessment of CAD burden and RNA-Seq experimental design}

To quantify CAD status, study subjects were assessed at enrolment for $\mathrm{CAC}$, using multi-slice computed tomography followed by Agatston scoring (see Methods). CAC scoring is a robust marker of CAD [15] and has been demonstrated to be useful for both calibration and discrimination of the disease burden. In addition, this measurement of CAD also has superior positive predictive value for future adverse cardiovascular events $[16,17]$. We selected eight age-, sex-, and ethnicitymatched case:control pairs from the extremes of the coronary calcium score distribution for combined RNA-Seq and microarray analysis and another eight matched case: control pairs for microarray-only analysis (Table 1). The median CAC scores for cases in the first and second groups were 1531.5 and 682.5, respectively. For comparison, even the presence of a CAC score (i.e., any non-zero value) is clinically considered indicative of CAD, while a score of 400 is often considered an advanced disease state [17]. When the median age for the cases in the first and second groups (56 and 61.5 years, respectively) and the ethnicity of these subjects (Caucasian) was considered, these scores corresponded to the $99^{\text {th }}$ and $93^{\text {rd }}$ centiles, respectively, as measured using the CAC score distribution from 6110 participants in the Multi-Ethnic Study of Atherosclerosis (MESA) [18]. This demonstrated the severity of CAD in our discovery cases. As part of the ClinSeq ${ }^{\bullet}$ protocol, all subjects were also analyzed using a set of 123 clinical chemistry tests and six phenotypic measurements (Additional file 2: Table S1). Results from these tests did not show significant association with calcification scores.

\section{Analyses of gene expression with RNA-Seq data}

To convert the RNA-Seq read data into a quantitative measure of gene expression, we calculated the number of RNA-Seq reads mapping to transcripts in the RefSeq database (see Methods). Such transcript-level read counts are better suited for statistical testing of gene expression differences compared to other RNA-Seq expression metrics [19]. Several statistical techniques have been 
Table 1 Clinical data for 32 subjects

\begin{tabular}{|c|c|c|c|c|c|}
\hline \multicolumn{3}{|c|}{ Controls } & \multicolumn{3}{|c|}{ Cases } \\
\hline ID & age & Calcification score & ID & age & Calcification score \\
\hline \multicolumn{6}{|c|}{ Discovery cohort (RNA-Seq + Microarray) } \\
\hline 112916 & 56 & 0 & 163383 & 55 & 1114 \\
\hline 116099 & 62 & 0 & 108971 & 63 & 1370 \\
\hline 119657 & 53 & 0 & 198564 & 53 & 1012 \\
\hline 133237 & 53 & 0 & 121424 & 52 & 514 \\
\hline 113235 & 53 & 0 & 165583 & 53 & 4352 \\
\hline 118655 & 58 & 0 & 189793 & 57 & 3532 \\
\hline 119522 & 65 & 0 & 158592 & 66 & 2885 \\
\hline 133871 & 56 & 0 & 132306 & 59 & 1693 \\
\hline \multicolumn{6}{|c|}{ Validation cohort (Microarray) } \\
\hline 199039 & 64 & 0 & 129854 & 64 & 1268 \\
\hline 124644 & 58 & 0 & 106945 & 58 & 890 \\
\hline 199794 & 58 & 0 & 116106 & 58 & 697 \\
\hline 102488 & 63 & 0 & 108828 & 64 & 693 \\
\hline 184917 & 57 & 0 & 155489 & 57 & 672 \\
\hline 154405 & 60 & 0 & 148238 & 61 & 668 \\
\hline 117532 & 62 & 0 & 142361 & 63 & 654 \\
\hline 192736 & 62 & 0 & 107143 & 62 & 565 \\
\hline
\end{tabular}

All subjects were males of Caucasian ethnicity.

developed for quantifying differential expression from RNA-Seq count data. To reduce artifacts arising from an arbitrary choice of any single technique, we used a dual analysis approach. First, we analyzed the count data with the Bioconductor package edgeR [20], which uses the negative binomial distribution to model the count data. Using the common dispersion setting in edgeR, we found 978 transcripts to be differentially expressed transcripts $(\mathrm{p}<0.05)$, of which 55 were significant after applying a 5\% Benjamini-Hochberg correction for multiple testing (Figure 1A). However, edgeR and other RNA-Seq analysis tools using the negative binomial distribution have high rates of false discovery [21]. To reduce artifacts arising from an arbitrary choice of any single technique, we applied a second, more conservative test (one-way ANOVA) (Figure 1B) in addition to edgeR, which returned 515 transcripts at $\mathrm{p}<0.05$ without correction for multiple testing. Overall, results from both techniques showed good agreement, although edgeR was more liberal than the ANOVA test in assigning low significance values. To define a gene list, we selected transcripts that were significant at $\mathrm{p}<0.05$ in both the edgeR and ANOVA results, retaining 186 transcripts from 116 genes (Additional file 2: Table S2). We noted here that neither edgeR nor ANOVA tests were designed for isoform-level quantification; hence,
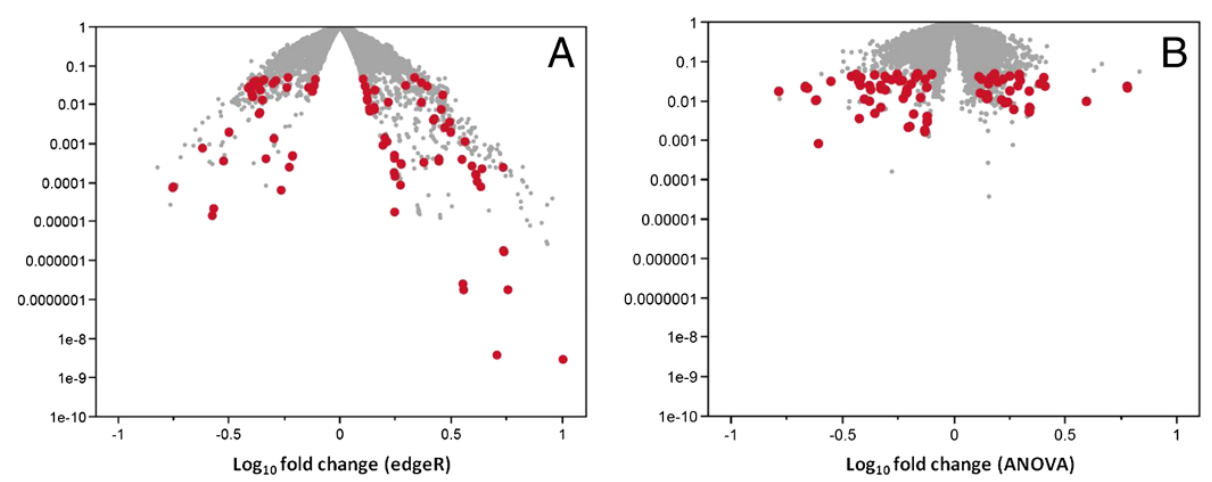

Figure 1 Volcano plots from edgeR (panel A) and ANOVA (panel B) analyses of RNA-Seq count data. X-axis on both panels shows base 10 logarithm of fold change (case/control). Y axis shows $p$ value. Red dots indicate 186 transcripts meeting $p<0.05$ in both tests. 
for genes with multiple transcripts in this list of 186, we could not identify which isoform was differentially expressed.

An interesting feature of the RNA-Seq results was that many transcripts expressed at very low levels showed significant differences between cases and controls. As genes expressed at near-background levels have historically been problematic for microarray technology [22], this aspect of the CAD transcriptome may benefit greatly from the increased dynamic range of RNA-Seq. To quantify absolute expression of these rare transcripts, we conducted a separate RNA-Seq experiment, starting with the same amount of total RNA used for other experiments in this study, with the difference being that the number of cells used for RNA extraction was precisely quantified. To the input RNA, we added seven polyadenylated non-mammalian RNA spikes, quantified such that their abundance (measured as copies per cell) spanned five orders of magnitude (Additional file 2: Table S3). Next, after sequencing this library to the same depth as the others ( 40 million reads), we converted RNA-Seq reads counts of these spikes to RPKM (Reads Per Million per Kilobase) values [19]. Finally, by comparing RPKM values of differentially expressed transcripts in our study with the spike-in control experiment, we determined that the cellular abundance of the rarer transcripts in our data approaches $\sim 5$ copies per cell (Figure 2). As many of the candidate genes with the highest statistical significance belong to this low-expression category (including LOC100131347 which had the lowest $\mathrm{p}$ value in our ANOVA results), some observations are relevant to note here. Firstly, before statistical testing, we screened out transcripts which had minimal or no coverage (see Additional file 1). Hence, these results are unlikely to represent statistical artifacts arising from low RNA-Seq coverage. Regarding quantity of input material, ten micrograms of total RNA were used for all RNA-Seq experiments, which is far above the minimum requirement for the Illumina RNA-Seq protocol. Consequently, paucity of input RNA should not cause transcripts to appear "rare" if they are otherwise expressed abundantly. Finally, each sample was sequenced using two full lanes of an Illumina flow cell for a relatively high depth of 40 million reads, which suggests that lack of sequencing coverage would not bias our results. Overall, these findings suggest that low-expression transcripts (many of which may have escaped detection with previous techniques) constitute an unexplored area of the CAD transcriptome that may contain clinically relevant information.

\section{Validation of RNA-Seq results using affymetrix arrays}

To address the effects of variation introduced by experimental procedures, we repeated the cell culture and

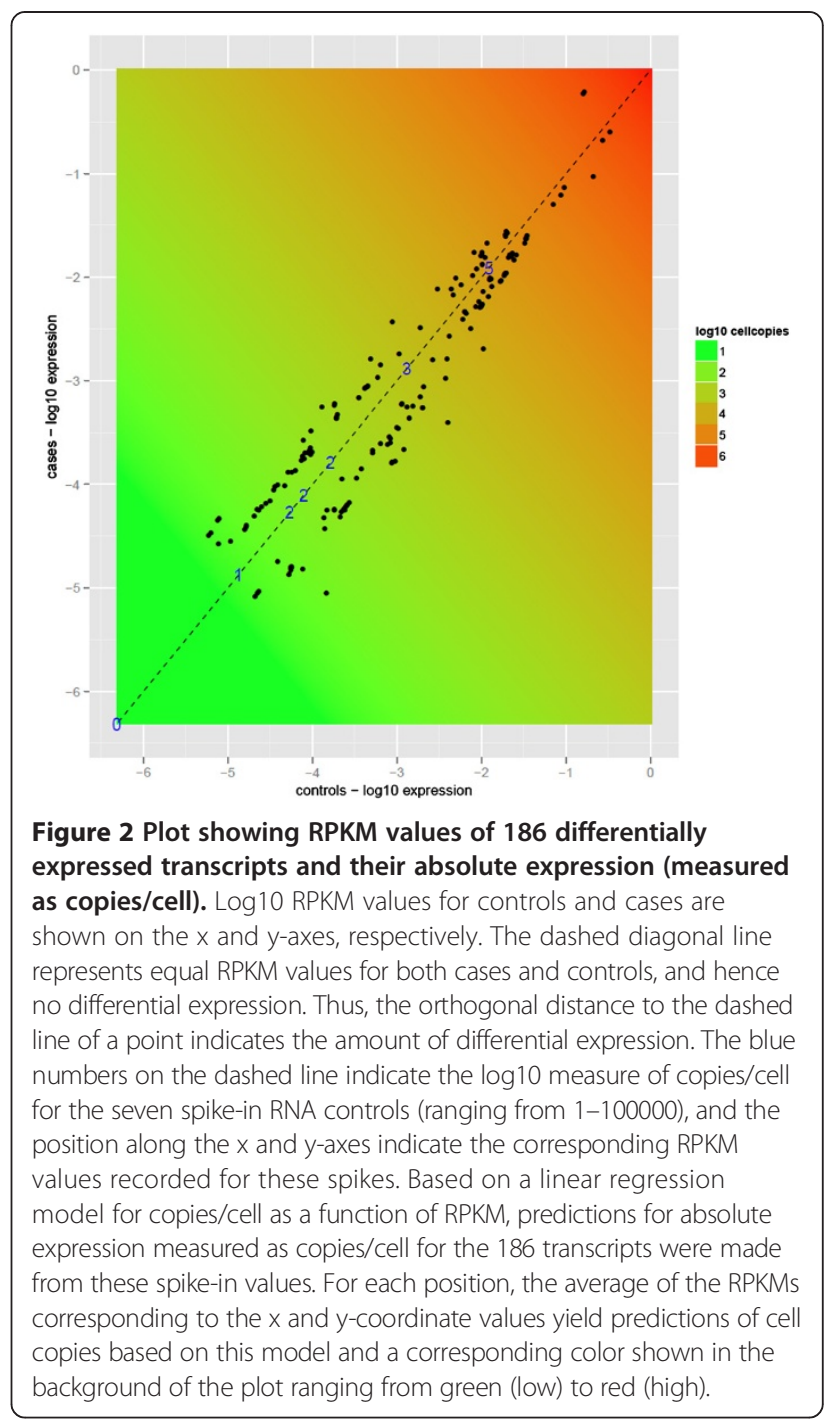

RNA extraction steps for all 16 cell lines used for the RNA-Seq, starting from previously frozen stocks. We then measured gene expression in these newer cultures using Affymetrix Human Exon 1.0 ST microarrays. Previous studies have demonstrated that these two methods of expression measurement (RNA-Seq and microarray) show strong agreement when applied to the same RNA sample [23]. Here, despite the fact that two additional passages of the cell lines took place in between the separate experiments, comparisons of the sixteen pairs of RNA-Seq and microarray results from the same subjects still showed high correlation (average $\mathrm{R}=0.73, \mathrm{p}<0.001$ ) (Additional file 1: Figure S1). Hence, we conclude that in vitro procedures did not appear to cause significant variability in the gene expression profiles of the cell lines.

To identify gene expression differences in cases and controls that were robust to both experimental (i.e., cell culture) replication as well as measurement technique, (i.e., RNA-Seq or microarray), we next selected genes 
that showed consistent up- or downregulation in both experiments. From the list of 186 differentially expressed transcripts found by RNA-Seq, 161 were included in the microarray probe design. Of these, 110 were regulated in the same direction (i.e. showed consistent upregulation or downregulation) in both the microarray and RNASeq data (Figure 3A). Given that these two gene expression measurements were conducted independently and on different RNA extraction batches, the observed agreement in fold change for 110/161 genes is highly significant (one-tailed Fisher's Exact Test, $\mathrm{p}<0.0001$; Additional file 2: Table S4). Next, we selected a validation cohort of eight additional matched case:control pairs from the extremes of the CAC score distribution (Table 1) and analyzed these samples using Affymetrix Human Exon 1.0 ST microarrays. Despite the fact that cases in this cohort had a lower median calcification score compared to the first cohort (682.5 and 1521.5, respectively), we again found a significant number of transcripts (71 of the above 110) with consistent up- or downregulation in both cohorts (one-tailed Fisher's Exact Test, $\mathrm{p}=0.0014$; Figure 3B; Additional file 2: Table S5). We conclude that these transcripts may be potential CAC biomarkers, as their expression differences persist even though the extent of calcification differed substantially when comparing our first and second cohorts.

Finally, we reasoned that direct evidence of a causal relationship with CAD would be obtained if any transcripts from LCL-based analyses exhibited differential expression patterns within actual atherosclerotic lesions. To identify such genes, we downloaded publicly available microarray data (GEO accession number GSE28829) from a gene expression study of human carotid artery autopsy samples [24]. From these samples (which were part of the Maastricht Pathology Tissue Collection), RNA was collected from 13 arteries in early CAD states (pathological intimal thickening and intimal xanthoma) and from 16 advanced CAD lesions (thin or thick fibrous cap atheroma). The two groups of samples, which were clearly separated by Principal Components Analysis, showed an abundance of genes with highly significant differential expression (4039 genes with $\mathrm{p}<0.05$, one-way ANOVA test with $10 \%$ Benjamini-Hochberg FDR correction, Additional file 1: Figure S2). However, our aim here was not to study these samples themselves but to identify genes showing similar patterns in LCLs and atherosclerotic arteries. To identify such genes, we calculated fold changes of 20639 genes that were measured in both studies (i.e. RNA-Seq in our project and the Affymetrix U133 Plus 2.0 microarrays used for the autopsy RNA study). Next, we selected genes that had a fold change $\geq 1.25$ in the same direction in all three data sets (i.e. first and second sets of CAC subjects in our study and in the CAD autopsy samples). Two genes (MMP7 and IGLL5) met this criterion, of which, $M M P 7$ is especially notable due to its functional role in atherosclerotic plaque rupture

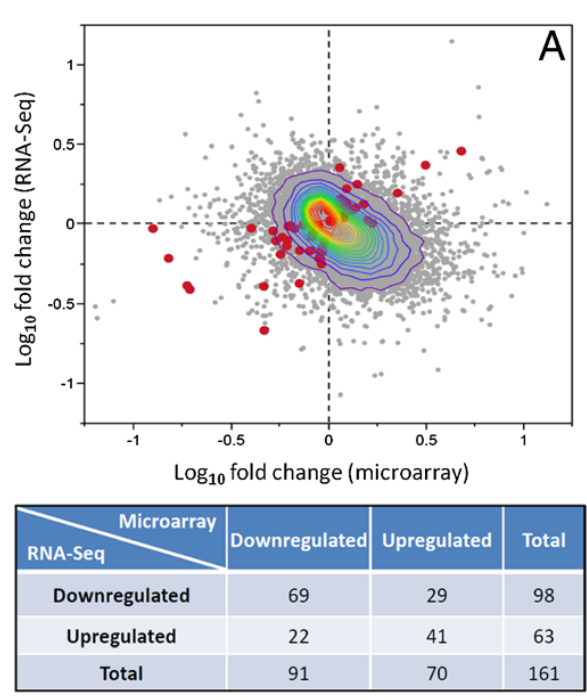

Two-tailed Fisher's Exact Test $\mathrm{p}<.0001$

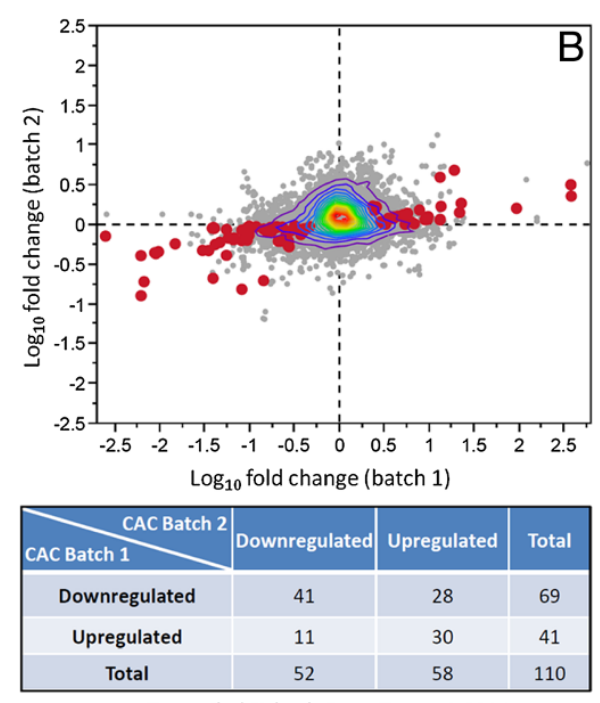

Two-tailed Fisher's Exact Test $\mathrm{p}=0.0014$

$\overline{1.2 .3 .4 .5 .6 .7 .8 .9}$ Quantile Density Contours

Figure 3 Validation of RNA-Seq results with microarray data. (A) Fold changes (case/control) from first 16 subjects, measured by RNA-Seq (Y-axis) and microarrays (X-axis) in logarithm base 10 scale. Red dots show 110 transcripts (out of 186 in Figure 1) that were upregulated or downregulated in both experiments. (B) Comparison of microarray data from first and second groups of 16 subjects (X-and Y-axes, respectively). Red dots show 71 transcripts out of 110 in panel A that were upregulated or downregulated in both groups. Contingency tables for statistical calculations are shown below each panel. 
and vascular smooth muscle cell apoptosis [25,26]. In addition, at the protein level, plasma concentrations of MMP7 were increased in patients with coronary artery disease [27]. Regarding IGLL5, this gene is interesting, as apart from being upregulated with disease in two unrelated tissues, it also had the largest fold change in the list of 186 significant transcripts. To investigate if IGLL5 is successfully translated in the arterial wall and if the mRNA difference persists at the protein level, we conducted preliminary immunoblotting experiments on lysates from human carotid plaques. Using an antihuman polyclonal antibody (which we confirmed to be IGLL5-specific using siRNA knockdown experiments; Additional file 1: Figure S3A-B), we compared three samples each from areas exhibiting early and advanced CAD (diffuse intimal thickening and fibroatheroma, respectively). Corresponding to the mRNA upregulation, we found a trend of increased protein levels in lysates from advanced disease (Figure 4). IGLL5 was also detected at abundant levels in the supernatants of the above lysates, suggesting that it is a secreted protein (Additional file 1: Figure S3C-D). Although the trend of protein upregulation was not statistically significant in this small set of samples (Additional file 2: Table S6), given the complexity of CAD etiology, followup studies of this gene using a larger sample size may be worthwhile.

\section{Analyses of transcription at unannotated genomic regions}

Recent evidence from RNA-Seq studies has demonstrated that the human genome is pervasively transcribed [28]. Thus, it is possible that CAD-relevant expression differences may exist in unannotated parts of the genome. Such differences would be even more interesting, as previous gene expression studies of CAD using microarrays would have entirely missed these regions due to their absence in the probe design. To explore this aspect of the transcriptome, we used the Cufflinks suite of RNA-Seq analysis tools (see Methods) [14]. First, we assembled novel transcripts, which we defined as those not included in a combination of several previous annotations including predicted genes. Next, we used the Cuffdiff tool to measure differential expression in these novel transcripts. Finally, we manually inspected the results to confirm that they showed evidence for being putative transcripts. Using this approach, we found eight novel loci that show differential expression with high statistical significance $(\mathrm{p}<0.0001$ after Benjamini-Hochberg correction) (Table 2). In terms of transcript structure, five of these eight loci contain multiple regions of increased read coverage joined by spliced RNA-Seq reads that mapped following the GT-AG rule (Figure 5). This strongly suggested the presence of a canonical exonintron structure. Mapping these loci to the ENCODE

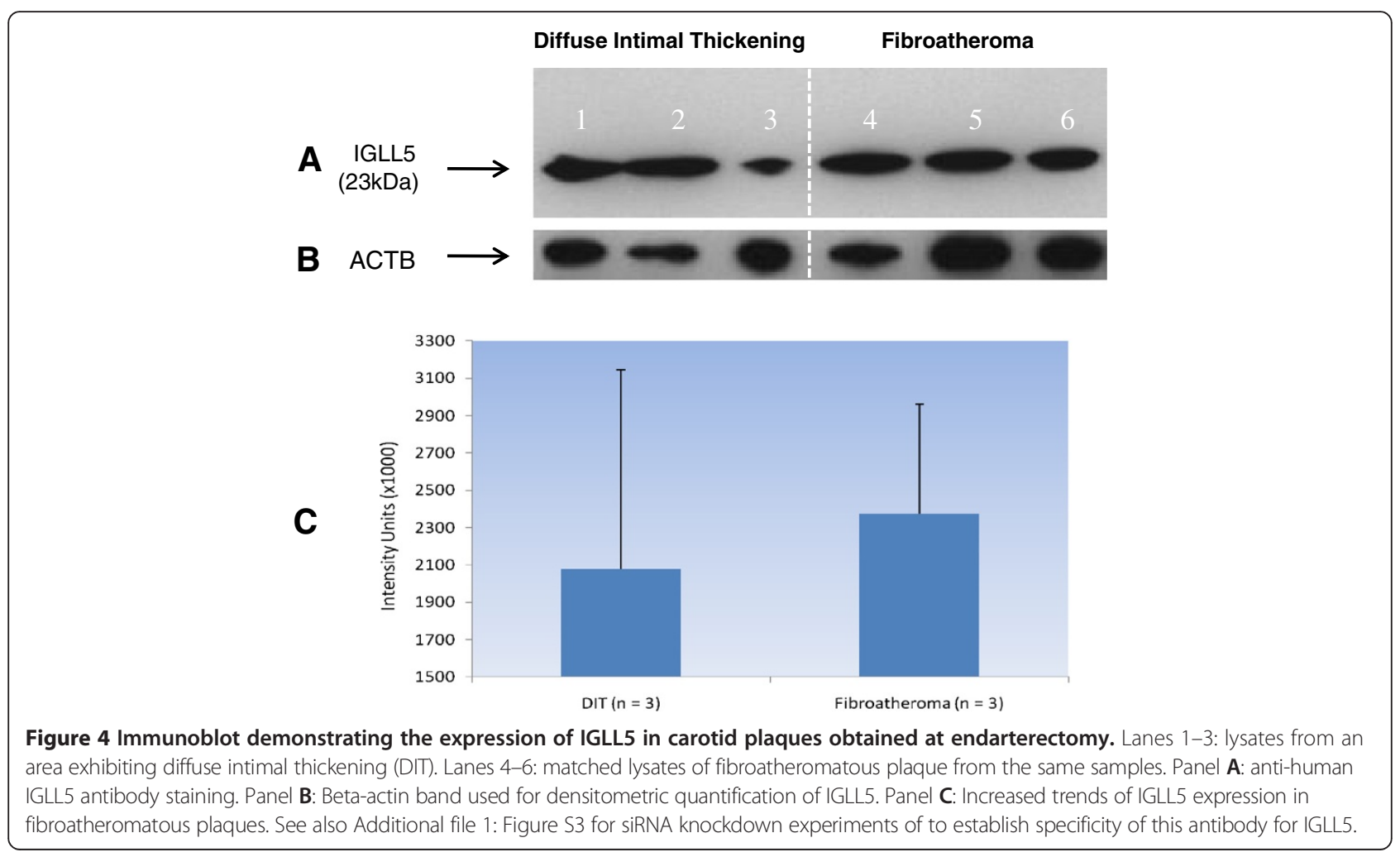


Table 2 Novel transcripts detected by Cufflinks/Cuffmerge/CuffDiff pipeline

\begin{tabular}{|c|c|c|c|c|c|c|}
\hline Name & Location & Fold change (case/control) & Corrected $\mathrm{p}$ value & Spliced? & Histone mark? & EST? \\
\hline CUFF.11349 & chr8:101524720-101588587 & -1.88185 & 0.041685 & Yes & Yes & Yes \\
\hline CUFF.2472 & chr12:31838565-31853772 & -2.26342 & 0.123977 & Yes & Yes & Yes \\
\hline CUFF.3 & chr1:80691-80824 & -11.5273 & 4.46E-08 & No & Yes & Yes \\
\hline CUFF.4 & chr1:452495-461228 & -10.6557 & $7.54 \mathrm{E}-10$ & Yes & Yes & Yes \\
\hline CUFF.5* & chr1:163653-164729 & -12.5567 & $1.96 \mathrm{E}-10$ & No & No & Yes \\
\hline CUFF.6* & chr1:164811-166159 & -12.8975 & $1.27 \mathrm{E}-12$ & No & No & No \\
\hline CUFF.7* & chr1:166235-166948 & -12.7567 & $1.27 \mathrm{E}-12$ & Yes & No & Yes \\
\hline CUFF.9 & chr1:227665-257075 & -11.1513 & $1.18 \mathrm{E}-08$ & Yes & Yes & Yes \\
\hline
\end{tabular}

Column 5 denotes presence of spliced reads following GT-AG rule. Column 6 denotes presence of layered H3K4Me1 or H3K4Me3 histone modification marks at the locus. Column 7 denotes the presence of spliced or unspliced human ESTs in the UCSC Genome Browser database. *CUFF.5, CUFF.6 and CUFF.7 were annotated by the Cufflinks pipeline as separate transcripts but their close location suggests that they may be components of the same transcript.

Integrated Regulation track on the UCSC Genome Browser showed histone modifications indicating active transcription. Although functional analyses will be required to confirm if these transcripts have genuine relevance to $C A D$, these results are interesting as they form the first description of unannotated regions of the transcriptome associated with this disease. As the ability to discover such novel transcripts is a significant advantage of RNA-Seq over existing techniques, we speculate that future analyses in different tissues and with larger sample sizes will continue to demonstrate that hidden components of CAD biology may exist outside known genes.

\section{Detection of candidate genes for alternative splicing}

The role of alternative splicing in CAD overall (or CAC in particular) has not been explored comprehensively, mainly due to the difficulties of microarray platforms in efficiently detecting changes in splicing patterns. In contrast, RNA-Seq is extremely powerful for studying

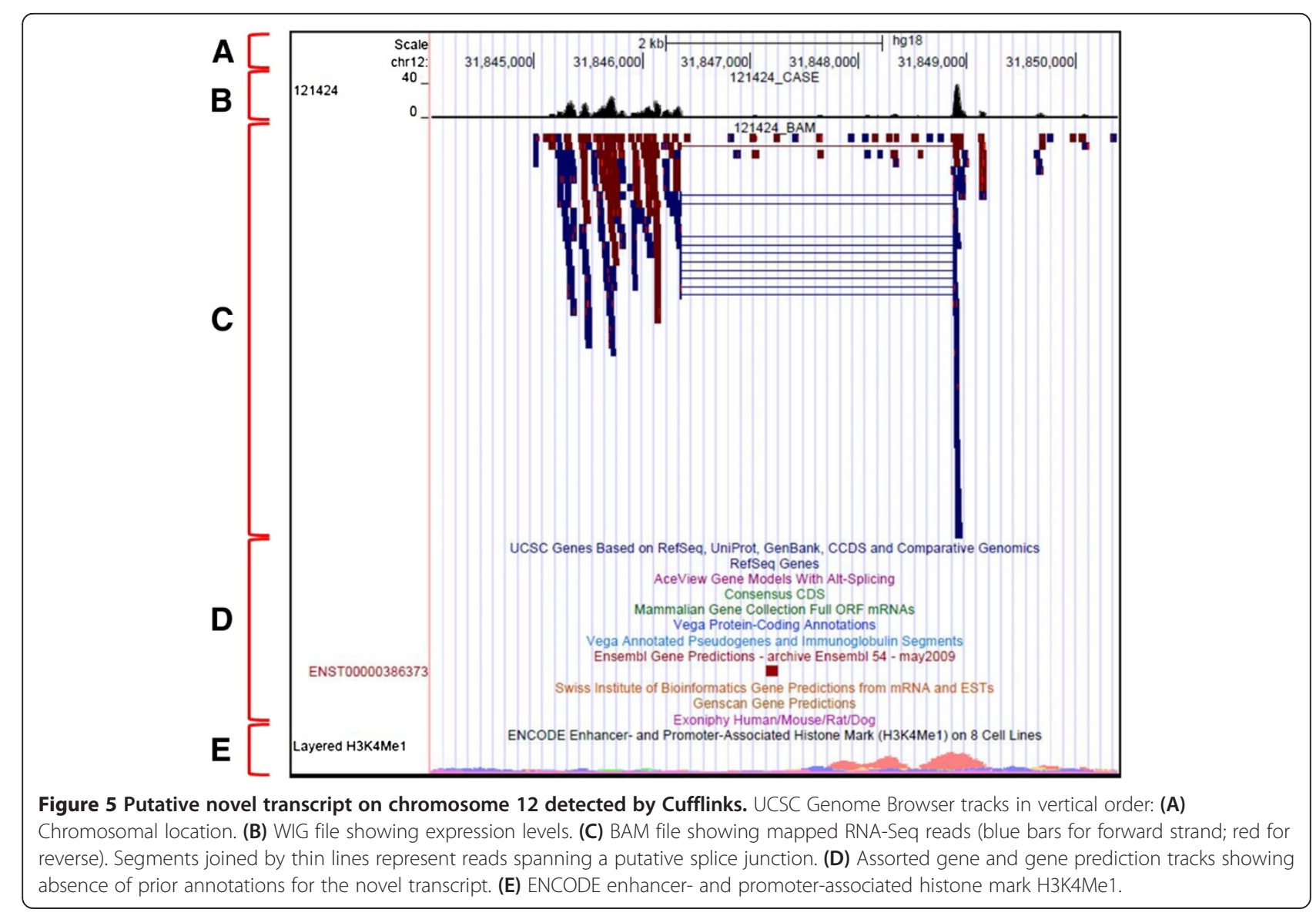


splicing variation. As statistical algorithms for such analyses are in an early stage of development, we chose to proceed with a conservative approach for detecting case:control splicing changes. Specifically, we analyzed exon-level RNA-Seq read counts using a linear ANOVA model fit to exon-level RNA-Seq read counts (see Methods). This model was designed to detect genes containing exons with increased case:control differential expression relative to other exons within the same transcript, which would thus be potential candidates for exon skipping, the most common form of alternative splicing. Using this approach, we detected 45 transcripts that show statistically significant differential usage in at least one exon ( $\mathrm{p} A C<0.05$ after $10 \%$ FDR correction) (Figure 6). These transcripts were then validated independently using the cuffdiff computational algorithm [14]. Eleven transcripts were validated by both methods and hence show promise for follow-up studies (Additional file 2: Tables S7 and S8). Interestingly, this list included the human leukocyte antigen gene $H L A-H$, variation in which has been associated with CAD susceptibility [29].

\section{Differentially expressed genes are enriched for CAD association and disease-relevant functions}

We next determined if differentially expressed genes showed an overall relationship with cardiovascular disease. To investigate this, we first combined previously compiled lists of CAD-associated genes [30,31]. From the combined list, we identified 3424 genes which showed expression in our RNA-Seq data. From this list, 43 genes are present in the list of 186 differentially expressed transcripts in our results. Statistically, this represented a highly significant enrichment of CAD-associated genes (onetailed Fisher's Exact Test, $\mathrm{p}=0.0002$; Additional file 1: Figure S4), suggesting that gene expression differences we detected by comparing cell lines from CAC cases and controls showed an overall relationship with CAD.

At the network level, differentially expressed genes may identify biological pathways that are perturbed in CAD. To investigate this hypothesis, we used Ingenuity Pathways Analysis $^{\mathrm{TM}}$ (IPA) software, which performs systems biology analysis using a large repository of previously documented gene-gene interactions and functional annotations (Additional file 3). The highest-scoring network (IPA score $=48$ ) in the list of 186 differentially expressed transcripts included the term "Cardiovascular System Development and Function", further highlighting the relationship of differential expression in this study with CAD. Interestingly, the second highest-scoring network (IPA score $=36$ ) contained the function "Lipid Metabolism", suggesting another possible mechanism through which gene expression differences may be related to CAD etiology. Finally, Gene Ontology (GO) term analysis of the 186 transcripts (using only LCL-expressed
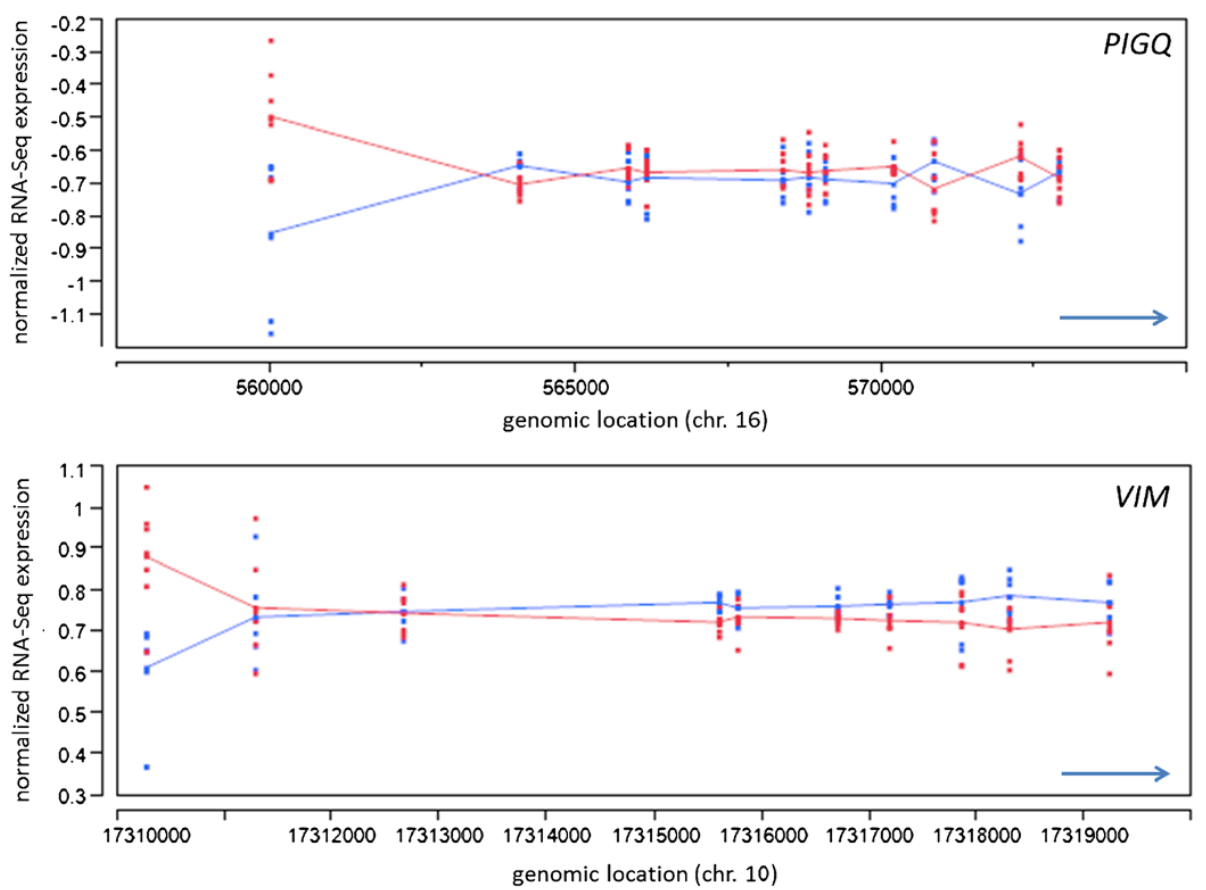

Figure 6 Examples of alternative splicing detected using exon-level analysis of RNA-Seq counts in PIGQ (top panel) and VIM (bottom panel). $X$ axis shows genomic location of exons (red and black dots for cases and controls, respectively) in hg18 coordinates. Y axis shows mean-subtracted normalized RNA-Seq read counts for each exon. Arrows at bottom right show direction of transcription. Neither gene was differentially expressed when all exons were considered together. 
genes as background to avoid bias), detected a number of networks related to cyclic AMP metabolism (Additional file 2: Table S9).

\section{Discussion}

We present here results from a pilot project for the use of RNA-Seq and cell lines in studying CAC gene expression. We used gene expression microarrays and cell culture replication for technical and experimental validation, respectively, of our initial results from this new technology. This project should be viewed in context of its scope and limitations; analyses of much larger numbers of LCLs will be required before any definitive statements can be made about the functional role in CAC of genes that we detected. However, our primary objective in this study was not functional analysis of such genes, but rather to identify candidate dysregulated genes in CAC using two relatively unexplored tools that may be useful overall in CAD research. By choosing LCLs from subjects who represent extreme outliers for the calcification score distribution, we hypothesized that between-group differences that are relevant for CAD may be detected. Evidence for this is reflected in the significant enrichment of CAD-associated genes in our results and further underscored in our IPA results, which show that differentially expressed genes we detected are concentrated in functional networks directly connected to CAD. However, we do not suggest that LCLs mirror in any way the transcriptional landscape of the atherosclerotic lesion itself. Rather, we hypothesize that our results represent eQTL-associated gene expression variants, which are stably propagated in these cell lines, underlying the observed differences between the case and control groups. We show that the combination of RNA-Seq and microarrays can be used to obtain useful results with a comparatively small sample size. In the near future, we anticipate that rapidly falling sequencing costs and development of user-friendly data analysis tools will soon make RNA-Seq the preferred alternative for transcriptome studies.

As the scope of this pilot project did not include functional validation assays, we refrain from speculating on the specific mechanisms by which individual genes may be involved in atherosclerosis development. However, as to the best of our knowledge, this study represents the first whole-transcriptome analysis specifically focusing on coronary artery calcification, and differentially genes that we found include many previous CAD candidates (such as MMP7, EPAS1, ESAM, CASP1, GUCY1A3, CLCN4, LEF1, ENPP5, and ZHX2), some observations may be relevant to state here. Interestingly, we detected differential expression of $Z H X 2$, a regulator of plasma lipid metabolism that is the closest gene to the top SNP locus from a recent atherosclerosis GWAS meta-analysis [32]. At the gene family level, the GIMAP family is enriched, with four genes (GIMAP1, GIMAP4, GIMAP5, and GIMAP7). Perhaps not by coincidence, multiple GIMAP family members have also been reported in a set of 128 genes (the "A-module") that is associated with atherosclerosis development. Overall, the GIMAP family has a crucial role in the development and function of $\mathrm{T}$ lymphocytes, which have an important role in the etiology of atherosclerosis [33]. Finally, it is intriguing that we detected differential expression of four genes involved in cAMP metabolism (ADM, APLP1, PRKCA and PTHLH), as existing studies focusing on individual genes involved in arterial calcification collectively suggest that perturbation of ATP metabolism plays a role in this process [34].

\section{Conclusions}

In summary, in this project we piloted the study of coronary artery calcification using cell lines as a patient surrogate for gene expression. We demonstrate that with careful experimental design and secondary validation of results, statistically significant results can be obtained with RNA-Seq even from a small sample size. In addition to canonical gene expression, we focused on alternative splicing and novel transcript discovery, two areas of the CAD transcriptome that may benefit the most from further RNA-Seq analyses. Studies using larger numbers of LCLs along with follow-up experiments will be needed to validate differentially expressed genes from this pilot study as true CAC biomarkers; this will become possible in the near future as we deploy RNA-Seq to the entire ClinSeq ${ }^{\circ}$ cohort.

\section{Additional files}

Additional file 1: Additional Methods and Figures Description: Expanded RNA-Seq protocol, statistical and bioinformatics methods. Additional file 1: Figures S1-S4.

Additional file 2: Additional Data. Description: Clinical data, expanded lists of differentially expressed and alternatively spliced transcripts and results from Western blotting.

Additional file 3: Ingenuity Pathways Analysis results Description: Complete output from Ingenuity Pathways Analysis.

\section{Abbreviations}

LCL: Lymphoblastoid cell line; EBV: Epstein-Barr virus; CAD: Coronary artery disease; CAC: Coronary artery calcification; GWAS: Genome-wide association study; FDR: False discovery rate.

\section{Competing interests}

Leslie G. Biesecker is an uncompensated consultant to the Illumina Corporation.

\section{Authors' contributions}

SKS, LGB, JCM, EDG and PJM designed the experiments. MYC and AEA carried out all $C T$ scanning and calcification scoring procedures. SL performed all cell culture experiments. SKS, DSA and NISC constructed and sequenced RNA-Seq libraries. AGE performed all microarray experiments. FDK, XZ and QC conducted all immunoblotting experiments. SKS, JJB, PFC, LNS, JCM and PJM participated in bioinformatics and statistical analysis of 
the RNA-Seq and microarray data. SKS and JJB drafted the manuscript. All authors read and approved the final manuscript.

\section{Acknowledgments}

The authors thank Peter Chines, Nancy Hansen, Jamie Teer, and Aaron Quinlan for computational analyses, Robert Blakesley, Alice Young, Michael Erdos, Marjorie Lindhurst, Jacquelyn Idol, and Jennifer Johnston for help with experimental procedures, Julia Fekecs for help with preparing high-resolution graphics, Soma Chowdhury for proofreading, and Flavia Facio, David Ng, Clesson Turner, and the ClinSeq ${ }^{\circledast}$ clinical support and nursing staff for their help with the clinical aspects of this study. We thank Eric Olivares and the SEQanswers community (http://SEQanswers.com) for providing valuable advice. Above all, we are grateful to the many participants of the ClinSeq ${ }^{\circledast}$ study. This study is supported by funds from the Intramural Research Program of the National Institutes of Health.

\section{Author details}

${ }^{1}$ National Human Genome Research Institute, National Institutes of Health, Bethesda, MD 20892, USA. ${ }^{2}$ Mathematical and Statistical Computing Laboratory, Center for Information Technology, National Institutes of Health, Bethesda, MD 20892, USA. ${ }^{3}$ CVPath Institute, Inc, Gaithersburg, MD 20878 , USA. ${ }^{4}$ National Heart Lung and Blood Institute, National Institutes of Health, Bethesda, MD 20892, USA.

Received: 11 October 2012 Accepted: 4 March 2014 Published: 14 March 2014

\section{References}

1. Koren O, Spor A, Felin J, Fåk F, Stombaugh J, Tremaroli V, Behre CJ, Knight $R$, Fagerberg B, Ley RE, Bäckhed F: Human oral, gut, and plaque microbiota in patients with atherosclerosis. Proc Natl Acad Sci USA 2011, 108(Suppl 1):4592-4598.

2. Lusis AJ, Mar R, Pajukanta P: Genetics of atherosclerosis. Annu Rev Genomics Hum Genet 2004, 5:189-218.

3. Roberts R, McPherson R, Stewart AFR: Genetics of atherosclerosis. In Cardiovascular Genetics and Genomics. Edited by Roden D. Oxford, UK: Wiley-Blackwell; 2009:151-166.

4. Bijnens AP, Lutgens E, Ayoubi T, Kuiper J, Horrevoets AJ, Daemen MJ: Genome-wide expression studies of atherosclerosis: critical issues in methodology, analysis, interpretation of transcriptomics data. Arterioscler Thromb Vasc Biol 2006, 26(6):1226-1235.

5. Skogsberg J, Lundstrom J, Kovacs A, Nilsson R, Noori P, Maleki S, Kohler M, Hamsten A, Tegner J, Bjorkegren J: Transcriptional profiling uncovers a network of cholesterol-responsive atherosclerosis target genes. PLOS Genet 2008, 4(3):e1000036.

6. Shendure J: The beginning of the end for microarrays? Nat Methods 2008, 5(7):585-587.

7. Greenland P, Alpert JS, Beller GA, Benjamin EJ, Budoff MJ, Fayad ZA, Foster E, Hlatky MA, Hodgson JM, Kushner FG, Lauer MS, Shaw LJ, Smith SC Jr, Taylor AJ, Weintraub WS, Wenger NK, Jacobs AK: 2010 ACCF/AHA guideline for assessment of cardiovascular risk in asymptomatic adults: executive summary: a report of the American College of Cardiology Foundation/American Heart Association Task Force on Practice Guidelines. Circulation 2010, 122(25):2748-2764.

8. Bullaughey $\mathrm{K}$, Chavarria Cl, Coop G, Gilad Y: Expression quantitative trait loci detected in cell lines are often present in primary tissues. Hum Mol Genet 2009, 18(22):4296-4303.

9. Veyrieras JB, Kudaravalli S, Kim SY, Dermitzakis ET, Gilad Y, Stephens M, Pritchard JK: High-resolution mapping of expression-QTLs yields insight into human gene regulation. PLOS Genet 2008, 4(10):e1000214.

10. Biesecker LG, Mullikin JC, Facio FM, Turner C, Cherukuri PF, Blakesley RW, Bouffard GG, Chines PS, Cruz P, Hansen NF, Teer JK, Maskeri B, Young AC, NISC Comparative Sequencing Program, Manolio TA, Wilson AF, Finkel T, Hwang P, Arai A, Remaley AT, Sachdev V, Shamburek R, Cannon RO, Green ED: The ClinSeq project: piloting large-scale genome sequencing for research in genomic medicine. Genome Res 2009, 19(9):1665-1674.

11. Agatston AS, Janowitz WR, Hildner FJ, Zusmer NR, Viamonte M Jr, Detrano R: Quantification of coronary artery calcium using ultrafast computed tomography. J Am Coll Cardiol 1990, 15(4):827-832.

12. Trapnell C, Pachter L, Salzberg SL: TopHat: discovering splice junctions with RNA-Seq. Bioinformatics 2009, 25(9):1105-1111.
13. Quinlan AR, Hall IM: BEDTools: a flexible suite of utilities for comparing genomic features. Bioinformatics 2010, 26(6):841-842.

14. Trapnell C, Williams BA, Pertea G, Mortazavi A, Kwan G, van Baren MJ, Salzberg SL, Wold BJ, Pachter L: Transcript assembly and quantification by RNA-Seq reveals unannotated transcripts and isoform switching during cell differentiation. Nat Biotechnol 2010, 28(5):511-515.

15. Greenland P, Alpert JS, Beller GA, Benjamin EJ, Budoff MJ, Fayad ZA, Foster E, Hlatky MA, Hodgson JM, Kushner FG, Lauer MS, Shaw LJ, Smith SC Jr, Taylor AJ, Weintraub WS, Wenger NK, Jacobs AK, Smith SC Jr, Anderson JL, Albert N, Buller CE, Creager MA, Ettinger SM, Guyton RA, Halperin JL, Hochman JS, Kushner FG, Nishimura R, Ohman EM, Page RL, et al: 2010 ACCF/AHA guideline for assessment of cardiovascular risk in asymptomatic adults: a report of the American College of Cardiology Foundation/American Heart Association Task Force on Practice Guidelines. J Am Coll Cardiol 2010, 56(25):e50-e103.

16. Folsom AR, Kronmal RA, Detrano RC, O'Leary DH, Bild DE, Bluemke DA, Budoff MJ, Liu K, Shea S, Szklo M, Tracy RP, Watson KE, Burke GL: Coronary artery calcification compared with carotid intima-media thickness in the prediction of cardiovascular disease incidence: the Multi-Ethnic Study of Atherosclerosis (MESA). Arch Intern Med 2008, 168(12):1333-1339.

17. Piers LH, Salachova F, Slart RH, Vliegenthart R, Dikkers R, Hospers FA, Bouma HR, Zeebregts CJ, Willems TP, Oudkerk M, Zijlstra F, Tio RA: The role of coronary artery calcification score in clinical practice. BMC Cardiovasc Disord 2008, 8:38.

18. McClelland RL, Chung H, Detrano R, Post W, Kronmal RA: Distribution of coronary artery calcium by race, gender, and age: results from the Multi-Ethnic Study of Atherosclerosis (MESA). Circulation 2006, 113(1):30-37.

19. Bullard $J \mathrm{H}$, Purdom $\mathrm{E}$, Hansen $\mathrm{KD}$, Dudoit $\mathrm{S}$ : Evaluation of statistical methods for normalization and differential expression in mRNA-Seq experiments. BMC Bioinforma 2010, 11:94.

20. Robinson MD, McCarthy DJ, Smyth GK: edgeR: a bioconductor package for differential expression analysis of digital gene expression data. Bioinformatics 2010, 26(1):139-140.

21. Tarazona S, Garcia-Alcalde F, Dopazo J, Ferrer A, Conesa A: Differential expression in RNA-seq: a matter of depth. Genome Res 2011, 21(12):2213-2223.

22. Canales RD, Luo Y, Willey JC, Austermiller B, Barbacioru CC, Boysen C, Hunkapiller K, Jensen RV, Knight CR, Lee KY, Ma Y, Maqsodi B, Papallo A, Peters EH, Poulter K, Ruppel PL, Samaha RR, Shi L, Yang W, Zhang L, Goodsaid FM: Evaluation of DNA microarray results with quantitative gene expression platforms. Nat Biotechnol 2006, 24(9):1115-1122.

23. Raghavachari N, Barb J, Yang Y, Liu P, Woodhouse K, Levy D, O'Donnell C, Munson PJ, Kato G: A systematic comparison and evaluation of high density exon arrays and RNA-seq technology used to unravel the peripheral blood transcriptome of sickle cell disease. BMC Med Genomics 2012, 5(1):28.

24. Gene Expression in early and advanced atherosclerotic plaque from human carotid. In [http://www.ncbi.nlm.nih.gov/geo/query/acc.cgi? acc $=$ GSE28829]

25. Halpert I, Sires UI, Roby JD, Potter-Perigo S, Wight TN, Shapiro SD, Welgus HD, Wickline SA, Parks WC: Matrilysin is expressed by lipid-laden macrophages at sites of potential rupture in atherosclerotic lesions and localizes to areas of versican deposition, a proteoglycan substrate for the enzyme. Proc Natl Acad Sci USA 1996, 93:9748-9753.

26. Williams H, Johnson JL, Jackson CL, White SJ, George SJ: MMP-7 mediates cleavage of $\mathrm{N}$-cadherin and promotes smooth muscle cell apoptosis. Cardiovasc Res 2010, 87(1):137-146.

27. Nilsson L, Jonasson L, Nijm J, Hamsten A, Eriksson P: Increased plasma concentration of matrix metalloproteinase-7 in patients with coronary artery disease. Clin Chem 2006, 52(8):1522-1527.

28. Clark MB, Amaral PP, Schlesinger FJ, Dinger ME, Taft RJ, Rinn JL, Ponting CP, Stadler PF, Morris KV, Morillon A, Rozowsky JS, Gerstein MB, Wahlestedt C, Hayashizaki $Y$, Carninci $P$, Gingeras TR, Mattick JS: The reality of pervasive transcription. PLOS Biol 2011, 9(7):e1000625-e1001102. discussion.

29. Takeuchi F, Yokota M, Yamamoto K, Nakashima E, Katsuya T, Asano H, Isono M, Nabika T, Sugiyama T, Fujioka A, Awata N, Ohnaka K, Nakatochi M, Kitajima H, Rakugi H, Nakamura J, Ohkubo T, Imai Y, Shimamoto K, Yamori Y, Yamaguchi S, Kobayashi S, Takayanagi R, Ogihara T, Kato N: Genome-wide association study of coronary artery disease in the Japanese. Eur J Hum Genet 2012, 20(3):333-340.

30. Hägg S, Skogsberg J, Lundström J, Noori P, Nilsson R, Zhong H, Maleki S, Shang MM, Brinne B, Bradshaw M, Bajic VB, Samnegård A, Silveira A, Kaplan 
LM, Gigante B, Leander K, de Faire U, Rosfors S, Lockowandt U, Liska J, Konrad P, Takolander R, Franco-Cereceda A, Schadt EE, Ivert T, Hamsten A, Tegnér J, Björkegren J: Multi-organ expression profiling uncovers a gene module in coronary artery disease involving transendothelial migration of leukocytes and LIM domain binding 2: the Stockholm Atherosclerosis Gene Expression (STAGE) study. PLoS Genet 2009, 5(12):e1000754.

31. Illumina HumanCVD Genotyping BeadChip. In [http://www.illumina.com/ documents/products/datasheets/datasheet humancvd.pdf

32. Bis JC, Kavousi M, Franceschini N, Isaacs A, Abecasis GR, Schminke U, Post WS, Smith AV, Cupples LA, Markus HS, Schmidt R, Huffman JE, Lehtimäki T, Baumert J, Münzel T, Heckbert SR, Dehghan A, North K, Oostra B, Bevan S, Stoegerer EM, Hayward C, Raitakari O, Meisinger C, Schillert A, Sanna S, Völzke H, Cheng YC, Thorsson B, Fox CS, et al: Meta-analysis of genome-wide association studies from the CHARGE consortium identifies common variants associated with carotid intima media thickness and plaque. Nat Genet 2011, 43(10):940-947.

33. Robertson AK, Hansson GK: T cells in atherogenesis: for better or for worse? Arterioscler Thromb Vasc Biol 2006, 26(11):2421-2432.

34. Rutsch F, Nitschke $Y$, Terkeltaub R: Genetics in arterial calcification: pieces of a puzzle and cogs in a wheel. Circ Res 2011, 109(5):578-592.

doi:10.1186/1471-2164-15-198

Cite this article as: Sen et al.: Identification of candidate genes involved in coronary artery calcification by transcriptome sequencing of cell lines. BMC Genomics 2014 15:198.

\section{Submit your next manuscript to BioMed Central and take full advantage of:}

- Convenient online submission

- Thorough peer review

- No space constraints or color figure charges

- Immediate publication on acceptance

- Inclusion in PubMed, CAS, Scopus and Google Scholar

- Research which is freely available for redistribution 